\title{
THE SEPARATION OF THE HAMILTON-JACOBI EQUATION FOR THE KERR METRIC
}

\author{
G. E. PRINCE ${ }^{1}$, J. E. ALDRIDGE ${ }^{1}$, S. E. GODFREY ${ }^{1}$ and G. B. BYRNES ${ }^{1}$
}

(Received 13 February 1995)

\begin{abstract}
We discuss the separability of the Hamilton-Jacobi equation for the Kerr metric. We use a recent theorem which says that a completely integrable geodesic equation has a fully separable Hamilton-Jacobi equation if and only if the Lagrangian is a composite of the involutive first integrals. We also discuss the physical significance of Carter's fourth constant in terms of the symplectic reduction of the Schwarzschild metric via $S O(3)$, showing that the Killing tensor quantity is the remnant of the square of angular momentum.
\end{abstract}

\section{Introduction}

Our objective is to provide a partial explanation for the separability of the HamiltonianJacobi equation for the Kerr metric in general relativity. We recognize that what is a satisfactory explanation to one reader is uninformative to another, so we will qualify our objective by saying that our analysis is based upon the Lagrangian formulation of the geodesic equations and the Hamilton-Jacobi theorem, and that it is centred upon a theorem which guarantees full separability of the Hamilton-Jacobi equation when the geodesic equations are completely integrable. (In the general, non-geodesic case, a completely integrable Lagrangian system will not have a fully separable HamiltonJacobi equation.) The feature of the Kerr metric that we hope to clarify is the relation between the four involutive integrals produced by the Hamilton-Jacobi separability and the separable co-ordinates themselves. We will show that the appearance of the quadratic integral means that these will not be coordinates for the spacetime $M$, but for submanifolds of $\mathbb{R} \times T M$. We will also briefly explain the relationship of the quadratic integral with the residual symmetry group after reduction by $S O(3)$ of the Schwarzschild evolution space.

\footnotetext{
'Department of Mathematics, La Trobe University, Bundoora, Victoria 3083, Australia.
}

(C) Australian Mathematical Society 1999, Serial-fee code 0334-2700/99 
The paper is laid out as follows: Section 2 contains a summary of the evolution space description of second order ordinary differential equations and, in particular, of geodesic equations. Section 3 covers the Hamilton-Jacobi equation and its separability, both in the general and geodesic cases. Section 4 deals specifically with the separability properties for the Kerr metric and Section 5 discusses the interpretation of Carter's constant. In Section 6 we consider open questions and relations with known results.

Our main reference is the recent paper by Prince, Aldridge and Byrnes [8], where the theorem for necessary and sufficient conditions on Hamilton-Jacobi separability is presented. Our tangent bundle approach to ordinary differential equations can be found in $[4,5]$ and [12]. The application of these techniques to the geodesic equations and their symmetries is performed in [10] and [11]. Woodhouse's treatment of Hamilton-Jacobi separability $([13,14])$ has been influential in our thinking as has the work of Benenti and Francaviglia (see the review [1]).

\section{Preliminaries}

We refer the reader to [10] and [11] for details of the Euler-Lagrange formulation of the geodesic equations involving the Poincaré-Cartan form. We will now give a brief account.

The spacetime $(M, g)$ has generic local coordinates $\left(x^{a}\right) . \mathbb{B} \times M$ (the graph space for geodesics) has local coordinates $\left(s, x^{a}\right)$. The evolution space is $E:=\mathbb{R} \times T M$ with associated local coordinates $\left(s, x^{a}, u^{a}\right)$. The geodesic Lagrangian, $L: E \rightarrow \mathbb{R}$, is given by

$$
L(s, x, u):=\frac{1}{2} g_{x}(u, u)=\frac{1}{2} g_{a b} u^{a} u^{b} .
$$

The Poincaré-Cartan form, $\theta_{L} \in \bigwedge^{\prime}(E)$, is just the pullback by the Legendre transformation of the Hamilton-Poincaré form $p_{a} d q^{a}-H d s$. In local coordinates it is

$$
\theta_{L}=L d s+g_{a b} u^{b} \theta^{a},
$$

where $\theta^{a}:=d x^{a}-u^{a} d t$ are the contact one-forms on $E$. Its exterior derivative is

$$
d \theta_{L}=g_{a b} \psi^{a} \wedge \theta^{b}
$$

where $\psi^{a}:=d u^{a}+\Gamma_{b c}^{a} u^{c} d x^{b}$. The two-form $d \theta_{L}$ is of maximal rank and its one dimensional kernel is spanned by the vector field

$$
\Gamma:=\frac{\partial}{\partial s}+u^{a} \frac{\partial}{\partial x^{a}}-\Gamma_{b c}^{a} u^{b} u^{c} \frac{\partial}{\partial u^{a}} .
$$


This is a second order differential equation field on $E$ because its integral curves are parametrised by $s$ and are solutions of the differential equations

$$
\dot{x}^{a}=u^{a}, \dot{u}^{a}=F^{a},
$$

where the $F^{a}$ are (in general) functions on $E$ of all $2 n+1$ variables. In our geodesic case $F^{a}:=\Gamma_{b c}^{a} u^{b} u^{c}$. In fact, the integral curves of $\Gamma$ are just the natural lifts from $M$ to $E$ of the solution curves of the geodesic equations. This is, of course, a direct result of the variational principle for $\theta_{L}$.

Now we turn to a brief discussion of symmetries. What follows holds for any (not necessarily autonomous) second order differential equation field $\Gamma$ on the evolution space $E$ of an arbitrary manifold $M$ of dimension $n$ with independent variable $t . E$ will have generic natural coordinates $\left(t, x^{a}, u^{a}\right)$. We refer the reader to [5] and [12] for details.

A $\Gamma$-basic form (or invariant form of Cartan [2]) is a form $\alpha$ on $E$ which satisfies any one of three equivalent conditions:

1. $\alpha(\Gamma)=0$ and $\Gamma\lrcorner d \alpha=0$.

2. $\mathscr{L}_{f \Gamma} \alpha=0$ for any smooth function $f$ on $E$.

3. $\alpha$ is the pullback of a form from the quotient of $E$ by the action of $\Gamma$.

The closed, $\Gamma$-basic 1-forms are locally just the exterior derivatives of first integrals of $\Gamma$.

A symmetry of the differential equation is a local one-parameter Lie group action on $E$ which permutes the integral curves of $\Gamma$ and, by projection, the solution curves on the base $M$ (although this induced action will not in general be that of a Lie group on $M$ ). If an action is generated by a vector field $X$ on $E$ then it is a symmetry of $\Gamma$ if and only if $\mathscr{L}_{X} \Gamma=\lambda \Gamma$ for some $\lambda \in C^{\infty}(E)$. Consequently, the symmetries of $\Gamma$ do not form a module over $C^{\infty}(E)$, but over the first integrals of $\Gamma$ and this module is closed under the Lie bracket. However, because any $C^{\infty}(E)$-multiple of $\Gamma$ is a trivial symmetry of $\Gamma$, addition of such multiples to generators of symmetries produces further symmetry generators. The images of the integral curves of $\Gamma$ under the original and modified actions differ only by parametrisation. For this reason we define a transverse field, $[X]$, to be the equivalence class of symmetries differing from $X$ by the addition of a $C^{\infty}(E)$-multiple of $\Gamma$. As an example of selection from the equivalence class, we could choose $\hat{X} \in[X]$ so that $\hat{X}(t)=0$ by setting $\hat{X}=X-X(t) \Gamma$, with $X$ any element of $[X]$. There is an important one-to-one correspondence between transverse fields on $E$ and vector fields on the quotient of $E$ by the action of $\Gamma$. This results from the identification of $[X]$ with the representative $X \in[X]$ which passes to the quotient (this $X$ generates an action leaving the parametrisation of the integral curves of $\Gamma$ unchanged because $\mathscr{L}_{X} \Gamma=0$ ).

Now suppose that we have a regular, non-zero (but not necessarily a free-particle) Lagrangian on $E$. (Given a regular Lagrangian, bounded below on $E$, we can always 
add a constant so as to make it non-zero.) The Poincaré-Cartan form in this general case has coordinate representation

$$
\theta_{L}=L d t+\frac{\partial L}{\partial u^{a}}\left(d x^{a}-u^{a} d t\right) .
$$

The second order differential equation field resulting from the Euler-Lagrange equations will be called $\Gamma$, that is,

$$
\Gamma\lrcorner d \theta_{L}=0, \quad \Gamma(t)=1 .
$$

It is shown in Proposition 1 of [9] that symmetries of $d \theta_{L}$ are also symmetries of $\Gamma$ (but the converse is not true). The generators of such symmetries satisfy $\mathscr{L}_{X} d \theta_{L}=0$ as do all other elements of their transverse field. It will be important for us to consider those elements $X$ with $\theta_{L}(X)=0$ (Proposition 1 of [9] guarantees the existence of this element). Because $L$ is regular, $d \theta_{L}$ has maximal rank and the 2 -form $d \theta_{L}$ provides a bijection $X \mapsto X\lrcorner d \theta_{L}$ between those vector fields on $E$ satisfying $\mathscr{L}_{X} d \theta_{L}=0$, $\theta_{L}(X)=0$ and closed, $\Gamma$-basic 1 -forms. This well-known result is a special case of the Noether-Cartan theorem (see [9] for a proof).

THEOREM (Noether-Cartan). The map $[X] \mapsto X\lrcorner d \theta_{L}$ is a bijection of symmetries of $d \theta_{L}$ (that is, $\mathscr{L}_{X} d \theta_{L}=0$ ) to closed basic 1-forms for $\Gamma$.

We will restrict ourselves to the bijection $\left.\Theta_{L}: X \mapsto X\right\lrcorner d \theta_{L}$ between symmetries of $d \theta_{L}$ with $\theta_{L}(X)=0$ and closed $\Gamma$-basic 1 -forms. We can use this map to define involutive first integrals in a natural way which corresponds to the Poisson bracket involution of Hamiltonian mechanics: two smooth first integrals $f, g$ on $E$ with $d f \wedge d g \neq 0$ on $E$ are said to be in involution with respect to $L$ if $d \theta_{L}\left(X_{f}, X_{g}\right)=0$. Here $X_{f}:=\Theta_{L}^{-1}(d f)$ and $X_{g}:=\Theta_{L}^{-1}(d g)$, that is, for example, $\mathscr{L}_{X_{f}} d \theta_{L}=0$, $\theta_{L}\left(X_{f}\right)=0$ and $\left.d f=X_{f}\right\lrcorner d \theta_{L}$. We remark that the map $(f, g) \mapsto-d \theta_{L}\left(X_{f}, X_{g}\right)$ does not turn $E$ into a Poisson manifold as it is only well-defined on first integrals. However, it does turn the quotient of $E$ by $\Gamma$ into a symplectic manifold.

The important properties of involutive first integrals are given in the following propositions from [9]:

PROPOSITION 1. The following conditions are equivalent:

(a) $f$ and $g$ are involutive first integrals with respect to $L$,

(b) $X_{f}(g)=0=X_{g}(f)$,

(c) $\left[X_{f}, X_{g}\right]=0$.

PROPOSITION 2. If $f$ and $g$ are involutive first integrals and if $Y_{f} \in\left[X_{f}\right]$ and $Y_{g} \in\left[X_{g}\right]$ are defined by

$$
Y_{f}:=X_{f}-X_{f}(t) \Gamma \text { and } Y_{g}:=X_{g}-X_{g}(t) \Gamma
$$


then

$$
\left[Y_{f}, Y_{g}\right]=0 \text {. }
$$

We can now define a Lagrangian system to be completely integrable with respect to $L$ if there exist $n$ first integrals $f^{a}$ with $d f^{1} \wedge \cdots \wedge d f^{n} \neq 0$ which are in involution with respect to $L$.

\section{The Hamilton-Jacobi equation}

To define the Hamilton-Jacobi equation for the arbitrary Lagrangian system described above we will completely bypass the usual canonical transformation approach. Instead we will define it by the way it appears in the proof of the the Liouville-Arnol'd theorem (see [9]). Consider $n$ (local) first integrals $h^{a}$, not necessarily in involution, with $d h^{1} \wedge \cdots \wedge d h^{n} \neq 0$. Change coordinates from $\left(t, x^{a}, u^{a}\right)$ to $\left(t, x^{a}, h^{a}\right)$ by locally inverting the expressions for the $h^{a}$ in terms of the $u^{a}$ and set

$$
E_{c}:=\left\{v \in E \mid h^{a}(v)=c^{a}, a=1, \ldots, n\right\} .
$$

We denote the exterior derivative on these level sets by $d_{D}$ (really the foliated derivative so that $\mathrm{D}$ is an integrable distribution of dimension $(n+1)$ tangent to $E_{c}$; see [9] ). Now if $v \in E_{c}$ then $\theta_{L}(v) \in T_{v}^{*} E_{c}$ because $\theta_{L}$ has no $d h^{a}$ components in the coordinates $\left(t, x^{a}, h^{a}\right)$ or any other such coordinate map needed in an atlas for $E_{c}$. The HamiltonJacobi equation for a local function $G$ on $E$ is

$$
d_{D} G=\theta_{L} .
$$

(Note that $d_{D} G(v) \in T_{v}^{*} E_{c}$ for $v \in E_{c}$.)

This equation can be made less local by only asking that $\theta_{L}$ be closed on $E_{c}$, however the real restrictions lie with the level sets $E_{c}$ themselves. For the remainder of the paper all considerations are local in the sense that $\theta_{L}$ will be assumed exact on such level sets.

We define a pair $\left(\left\{h^{1}, \ldots, h^{n}\right\}, G\right)$ or equivalently $(D, G)$ to be a complete solution of the Hamilton-Jacobi equation if it satisfies

$$
d_{D} G=\theta_{L} .
$$

Proposition 3 below shows that the first integrals $h^{a}$ in a complete solution are in involution.

In the coordinates $\left(t, x^{a}, h^{a}\right)$ the Hamilton-Jacobi equation becomes

$$
L d t+\frac{\partial L}{\partial u^{a}}\left(d x^{a}-u^{a} d t\right)=\Gamma(G) d t+\frac{\partial G}{\partial x^{a}}\left(d x^{a}-u^{a} d t\right),
$$


where the $u^{a}$ are of course functions of $t, x^{a}$ and the values of the $h^{a}$ on each leaf. In the $\left(t, x^{a}, h^{a}\right)$ coordinates we have

$$
\Gamma=\frac{\partial}{\partial t}+u^{a} \frac{\partial}{\partial x^{a}}
$$

and the equations

$$
\Gamma(G)=L, \quad \frac{\partial G}{\partial x^{a}}=\frac{\partial L}{\partial u^{a}}
$$

are just the Hamilton-Jacobi equations pulled back to $\mathbb{R} \times T M$ from $\mathbb{R} \times T^{*} M$ by the Legendre transformation. Finding the function $G$ thus means finding a complete solution of the Hamilton-Jacobi equation.

The proof given in [9] of Liouville-Arnol'd theorem shows that complete integrability guarantees a complete solution of the Hamilton-Jacobi equation. We will now show that the inverse is also true.

Proposition 3. The existence of a complete solution of the Hamilton-Jacobi equation guarantees complete integrability.

ProOF. Let $G$ be a complete solution of the Hamilton-Jacobi equation. This implies the existence of $n$ local first integrals $h^{a}$ satisfying $d h^{1} \wedge \cdots \wedge d h^{n} \neq 0$ such that $d_{D} G=\theta_{L}$. So

$$
\begin{aligned}
d G & =\theta_{L}+\frac{\partial G}{\partial h^{a}} d h^{a} \\
\Rightarrow d \theta_{L} & =-d\left(\frac{\partial G}{\partial h^{a}} d h^{a}\right)=-d\left(\frac{\partial G}{\partial h^{a}}\right) \wedge d h^{a}
\end{aligned}
$$

in coordinates $\left(t, x^{a}, h^{a}\right)$ related by local inversion of the expressions for the $h^{a}$ to the coordinates $\left(t, x^{a}, u^{a}\right)$. Hence

$$
d \theta_{L}\left(X_{h^{a}}, X_{h^{b}}\right)=0
$$

and so the $h^{a}$ are in involution and the system is completely integrable.

From now on we will assume that our system is completely integrable with respect to $L$ and that the $n$ first integrals $f^{a}$ are in involution.

We now turn to the definitions of separability. It will be useful to adopt a notation for declaring functional dependency. Here is an example of what we will use: let $K$ be a function on $E$ with local coordinates $\left(t, x^{a}, u^{a}\right)$ then $K\left[t, u^{a}\right]$ will be used in place of $K$ to indicate that the coordinate representation of $K$ does not depend on the functions $x^{a}$. It will also be useful to have some generic coordinates $\left(\bar{x}^{0}, \bar{x}^{a}, f^{a}\right)$ for 
$E$. In general the maps $\bar{x}^{0}$ and $\bar{x}^{a}$ will not simply be composites of the maps $t$ and $x^{a}$ but will also involve the $f^{a}$ (and hence the $u^{a}$ ).

A complete solution $G$ of the Hamilton-Jacobi equation is separable with respect to coordinates $\left(\bar{x}^{0}, \bar{x}^{a}, f^{a}\right)$ if

$$
G\left[\bar{x}^{0}, \bar{x}^{a}, f^{a}\right]=G_{0}\left[\bar{x}^{0}, f^{a}\right]+\cdots+G_{n}\left[\bar{x}^{n}, f^{a}\right] .
$$

A complete solution $G$ of the Hamilton-Jacobi equation is fully separable with respect to coordinates $\left(\bar{x}^{0}, \bar{x}^{a}, f^{a}\right)$ if

$$
G\left[\bar{x}^{0}, \bar{x}^{a}, f^{a}\right]=G_{0}\left[\bar{x}^{0}, f^{a}\right]+\sum_{b=1}^{n} G_{b}\left[\bar{x}^{b}\right] f^{b} .
$$

The following lemma indicates the relationship between fully separable coordinates associated with a given set of involutive integrals. It is proved in [8].

LEMMA 1. If the Hamilton-Jacobi equation admits a solution of the form:

$$
G\left[\bar{x}^{0}, \bar{x}^{a}, f^{a}\right]=\bar{G}_{0}\left[\bar{x}^{0}, f^{a}\right]-f^{c} \bar{x}^{c}
$$

then it also admits a solution of the form

$$
G\left[y^{0}, y^{a}, f^{a}\right]=G_{0}\left[y^{0}, f^{a}\right]-f^{c} y^{c}
$$

in coordinates $\left(\bar{x}^{0}, y^{a}, f^{a}\right)$ where $\Gamma\left(G_{0}\right)=L$ and $\Gamma\left(y_{a}\right)=0$.

The $d t$-free Cartan symmetries corresponding to a set of involutive first integrals commute amongst themselves and with $\Gamma$, moreover they (along with $\Gamma$ ) form a basis for tangent spaces of the common level sets of the first integrals. Consequently, these $n+1$ fields are coordinate fields for the level sets. The obvious question then is whether or not these coordinates provide separability for the corresponding Hamilton-Jacobi equation.

The answer is generally no (the two dimensional Kepler problem is easily shown to be a counter-example). The same observations can be made for the $\theta_{L}-$ free Cartan symmetries and they also are inappropriate for the task ( $\theta_{L}$ annihilates all these fields). However, as it happens the appropriate coordinate fields, when they exist, are elements of the transverse fields of Cartan symmetries of the involutive integrals.

For the sake of brevity we will write $X_{a}:=\Theta_{L}^{-1}\left(d f^{a}\right)$ for each of our involutive integrals $f^{a}$. We will write $Y_{a}$ for the $d t$-free elements of $\left[X_{a}\right]$ described in Proposition 2. In the following theorem we look for $W_{a} \in\left[X_{a}\right]$ with $W_{a}=Y_{a}+\alpha_{a} \Gamma$ so that, along with $\Gamma$, these fields provide coordinate fields for coordinates $\left(y^{0}, y^{a}\right)$ on the level sets of the $f^{a}$ in which the solution of the Hamilton-Jacobi equation separates. 
THEOREM (Hamilton-Jacobi separability). The Hamilton-Jacobi equation admits a solution of the form:

$$
G\left[\bar{x}^{0}, \bar{x}^{a}, f^{b}\right]=\bar{G}_{0}\left[\bar{x}^{0}, f^{b}\right]-f^{a} \bar{x}^{a}
$$

if and only if

$$
\Gamma\left(\alpha_{a}\right)=0,
$$

where the $\alpha_{a}$ are defined by $\alpha_{a} L:=-f^{a}-\theta_{L}\left(Y_{a}\right)$.

The proof is given in [8].

COROLLARY 1. If the Hamilton-Jacobi equation has a solution of the form:

$$
G\left[y^{0}, y^{a}, f^{b}\right]=G_{0}\left[y^{0}, f^{b}\right]-f^{c} y^{c}
$$

then there exists an equivalent solution of the form

$$
\hat{G}\left[y^{0}, y^{a}, f^{b}\right]=\hat{G}_{0}\left[y^{0}\right]-f^{c} y^{c} .
$$

Again, we refer the reader to [8] for a proof.

The separability theorem takes on a simple form when the Lagrangian is itself a first integral, in particular when it is a composite of the involutive integrals. The following corollary shows that the Lagrangian is always a composite of the involutive integrals associated with a fully separable solution.

COROLlARY 2 (Geodesic motion). Let $\Gamma$ be the Euler field for geodesic motion on an n-dimensional (pseudo-) Riemannian manifold $M$ with Lagrangian $L . \quad$ If $\left\{f^{1}, \ldots, f^{n}\right\}$ are an involutive set with respect to $L$ then the Hamilton-Jacobi equation is fully separable with respect to this set if and only if $L$ is a composite of the $f^{a}$ 's.

PROOF. The condition of the separability theorem becomes $\Gamma\left(\alpha_{a} L\right)=0$ since $\Gamma(L)=0$ by assumption. Using $\alpha_{a} L=-f^{a}-\theta_{L}\left(Y^{a}\right)$ this condition is

$$
0=\Gamma\left(\theta_{L}\left(Y^{a}\right)\right)=\Gamma\left(Y^{a}(G)\right)=Y^{a}(\Gamma(L))=Y^{a}(L),
$$

where the second equality follows since the $Y^{a}$ are tangent to the level sets of the $f^{a}$ 's. Now $Y^{a}(L)=0$ for $a=1, \ldots, n$ if and only if $L$ is a composite of the $f^{a}$ 's because of the complete integrability and the assumption that $\Gamma(L)=0$. Hence the result follows. 
This result has broader applicability: suppose that $L$ is one of the $f^{a}$ 's and that we pose the question of separability with respect to the complementary involutive integrals, the $\frac{\partial G}{\partial f^{a}}$ 's (in the coordinates $\left(y^{0}, y^{a}, f^{a}\right)$ for example). Because $L$ is certainly not a composite of these complementary integrals the corollary tells us that the system is not separable with respect to these involutive integrals. The principle here is that complete separability is probably in general a function of the involutive integrals appearing in a given complete solution of the Hamilton-Jacobi equation. For this reason it is rather difficult to formulate results claiming complete separability or its absence based on the equations of motion alone.

\section{Hamilton-Jacobi separability for the Kerr metric}

Let $\mathbb{R} \times M$ be the extended configuration space of the Kerr spacetime $(M, g)$. We will use Boyer-Lindquist coordinates $\left(s, x^{0}, x^{1}, x^{2}, x^{3}\right):=(s, t, r, \theta, \phi)$ for $\mathbb{R} \times M$ where $s$ is the proper time (we make this choice for cosmetic reasons). The remaining natural coordinates for $\mathbb{R} \times T M$ are $\left(u^{a}\right)$.

The geodesic equations are completely integrable and the responsible involutive integrals are

$$
\begin{aligned}
f^{1}: & =\frac{1}{2} g_{a b} u^{a} u^{b}, \quad f^{3}:=-g_{3 a} u^{a}, \\
f^{2}: & =-g_{0 a} u^{a}, \quad f^{4}:=\frac{1}{2} K_{a b} u^{a} u^{b} .
\end{aligned}
$$

$f^{1}$ is just the geodesic Lagrangian $L$ and $K$ is Carter's Killing tensor [3]. The corresponding $d t$-free Cartan symmetries (see [10] and [11]) are

$$
\begin{array}{ll}
Y_{1}=\frac{\partial}{\partial s}-\Gamma, & Y_{3}=\frac{\partial}{\partial \phi}, \\
Y_{2}=\frac{\partial}{\partial t}, & Y_{4}=-K_{a}^{b} u^{a} \frac{\partial}{\partial x^{b}}-\Gamma\left(K_{a}^{b} u^{a}\right) \frac{\partial}{\partial u^{b}} .
\end{array}
$$

We can immediately apply Corollary 3 and say that the system is separable with respect to this involutive set because $f^{1}:=L$. It is a straightforward matter to show that the corresponding $\alpha$ 's are

$$
\alpha_{1}=1, \quad \alpha_{2}=0, \quad \alpha_{3}=0, \quad \alpha_{4}=\frac{f^{4}}{L}
$$

(which are indeed all constant along the integral curves of $\Gamma$ ) so that the separable coordinate fields for the level sets of the $f$ 's are

$$
\Gamma, \quad W^{1}:=\frac{\partial}{\partial s}, \quad W^{2}:=\frac{\partial}{\partial t}, \quad W^{3}:=\frac{\partial}{\partial \phi}, \quad W^{4}:=Y^{4}+\frac{f^{4}}{L} \Gamma .
$$


It is clear that these coordinates are not adapted to $\mathbb{R} \times T M$ and full separability is not achievable with coordinates from $\mathbb{R} \times M$ although Carter [3] shows that ordinary separability is possible. The relation between $\mathbb{R} \times M$ separability and $\mathbb{R} \times T M$ is discussed in general terms in [8]. We confess to not having computed the fully separable coordinates (this is done by computing the $\frac{\partial G}{\partial f^{a}}$ ) although it would be interesting to discuss the geodesic deviation in terms of the projected action of $W_{4}$. From the point of view of this paper the Kerr metric is important because it provides an example of a fully separable system with $\Gamma(L)=0$ which further has a hidden symmetry.

\section{Residual actions of $S O(3)$ and Carter's constant}

There is a large literature concerning the physical interpretation of Carter's Killing tensor conserved quantity for the Kerr metric. However, there appears to be nothing written relating the constant to a corresponding quantity for the Schwarzschild solution. In [6] the Marsden-Weinstein symplectic reduction procedure is performed on the Schwarzschild solution. Loosely, the procedure involves restricting the evolution space action to the common level set of some $m$ known first integrals; because the corresponding Cartan fields will generally not all be tangent to this level set we look for a residual symmetry group of dimension $r$, say, whose generators are tangent to the set. (See Section 6.3 of Olver [7].) These generators are symmetries of the (restricted) Cartan 2-form and so we have a reduced Lagrangian system with an $r$-dimensional symmetry group and the procedure is then iterated. In the case of the Schwarzschild metric, an initial choice of first integrals comprising $L$ and the linear integrals corresponding to coordinate time translation and rotations (five altogether) produces an Abelian residual symmetry group generated by $\frac{\partial}{\partial s}, \frac{\partial}{\partial t}, \frac{\partial}{\partial \phi}$ and the generator of rotations about the direction of conserved angular momentum. This last generator is $r^{2} \dot{\theta} \frac{\partial}{\partial \theta}+r^{2} \dot{\phi} \frac{\partial}{\partial \phi}$; this field is the Cartan symmetry of (the quadratic integral) $J^{2}$ so that the residual symmetry group corresponds to the energy, the linear coordinate time integral, the $z$-component of angular momentum and the square of angular momentum (three of which form the most celebrated set of integrals in mathematical physics). This Abelian group acts on the 4-dimensional common level sets of the 5 integrals and it gives a complete reduction of the problem at the next step (that is, a solution of the geodesic equations). (Of course, the system is completely integrable with respect to the four integrals corresponding to the residual symmetry group and so the Liouville-Arnol'd theorem can be used to obtain the remaining integrals needed.)

Now the Kerr metric does not enjoy $S O(3)$ invariance but the three symmetries $\frac{\partial}{\partial s}, \frac{\partial}{\partial t}, \frac{\partial}{\partial \phi}$ do commute and one might ask whether the Cartan symmetry for Carter's constant reduces to the generator of the square of angular momentum in the Schwarzschild limit. Godfrey [6] shows that this is the case. This is further evidence 
for the angular momentum at infinity interpretation of Carter's constant, but it also suggests that the separability of the Hamilton-Jacobi equation for the two problems are intimately related, with the failure of the Kerr metric to have fully separable coordinates on $\mathbb{R} \times M$ being due to the fact that the Cartan symmetry for the fourth constant is not the generator of a residual action of a group action on $\mathbb{R} \times M$ while this is true for the Schwarzschild case. Presumably the fully separable coordinates for the Kerr metric do reduce to the Schwarzschild ones in the limit; we leave this to the interested reader.

\section{Discussion}

The relationship of the results presented here to the important theorems of Woodhouse and Benenti is as yet unclear. Woodhouse [14] deduces the existence of local linear or quadratic first integrals from separability in just one spatial coordinate in the $L=T+U$ case. The best that we can do at the moment is to deduce from full separability (in our sense) and the existence of a coordinate field lifted from the base that a linear or quadratic integral exists (see [8]). The work of Benenti relates specifically to general relativity and the key theorem for us is the one which guarantees on an $n$-dimensional Riemannian manifold $r$ ignorable (and hence separable) coordinates on the spacetime if and only if there exist locally $r$ Killing vectors and $n-r$ Killing tensors which commute and are independent and moreover there exist $n-r$ independent, orthogonal, commuting common eigenvector fields of the Killing tensors which commute with the Killing vectors and are orthogonal to them (Theorem 2.7 of [1]). This theorem raises a number of questions: if the conditions of this theorem are satisfied (so that clearly the system is completely integrable) then what is the relationship between the non-projectable coordinate fields amongst our fully separable basis for $T E$ and the eigenvector fields of the theorem? Secondly, how does orthogonality appear in our result where the manifold is the evolution space? And finally, what role is played by the points in $E$ where the coordinate inversion from $\left(s, x^{a}, u^{a}\right)$ to $\left(s, x^{a}, f^{a}\right)$ cannot be performed?

We hope to address these questions in detail in the near future.

\section{References}

[1] S. Benenti and M. Francaviglia, "The theory of separability of the Hamilton-Jacobi equation and its applications to general relativity", in General Relativity and Gravitation, Volume 1 (ed. A. Held), (Plenum, N. Y., 1980).

[2] E. Cartan, Leçons sur les Invariants Intégraux (Hermann, Paris, 1922).

[3] B. Carter, "Global structure of the Kerr family of gravitational fields", Phys. Rev. 174 (1968) 1559-1571. 
[4] M. Crampin and F. A. E. Pirani, Applicable Differential Geometry (CUP, 1987).

[5] M. Crampin, G. E. Prince and G. Thompson, "A geometric version of the Helmholtz conditions in time dependent Lagrangian dynamics", J. Phys. A: Math. Gen. 17 (1984) 1437-1447.

[6] S. E. Godfrey, "Reduction of order techniques for classical orbit problems", Ph. D. Thesis, Department of Mathematics, La Trobe University, 1992.

[7] P. J. Olver, Applications of Lie Groups to differential Equations, second ed. (Springer-Verlag, 1993).

[8] G. E. Prince, J. E. Aldridge and G. B. Byrnes, "A universal Hamilton-Jacobi equation for secondorder ODEs", J. Phys. A: Math. Gen. 32 (1999) 827-844.

[9] G. E. Prince, G. B. Byrnes, J. Sherring and S. E. Godfrey, “A generalisation of the Liouville-Arnol'd theorem", Math. Proc. Camb. Phil. Soc. 117 (1995) 353-370.

[10] G. E. Prince and M. Crampin, "Projective differential geometry and geodesic conservation laws in general relativity, I: Projective actions", Gen. Rel. Grav. 16 (1984) 921-942.

[11] G. E. Prince and M. Crampin, "Projective differential geometry and geodesic conservation laws in general relativity, II: Conservation laws", Gen. Rel. Grav. 16 (1984) 1063-1075.

[12] W. Sarlet, G. E. Prince and M. Crampin, "Adjoint symmetries for time dependent second order equations", J. Phys. A: Math. Gen. 23 (1990) 1335-1347.

[13] N. Woodhouse, "Killing tensors and the separability of the Hamilton-Jacobi equation", Commun. Math. Phys 44 (1975) 9-38.

[14] N. Woodhouse, Geometric Quantisation, second ed. (OUP, 1992). 